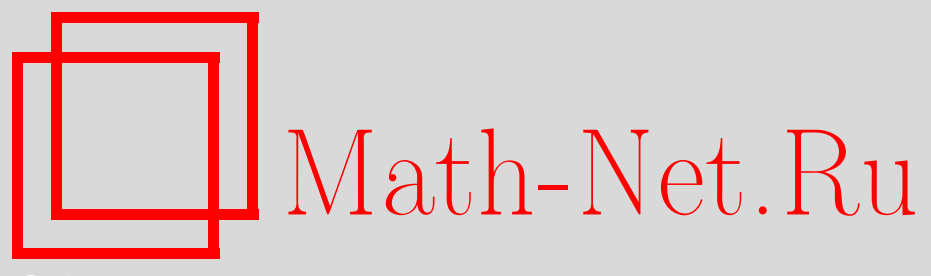

Н. Ю. Ероховец, Момент-угол многообразия простых $n$ мерных многогранников с $n+3$ гипергранями, $У M H$, 2011, том 66, выпуск 5, 187-188

DOI: https://doi.org/10.4213/rm9446

Использование Общероссийского математического портала Math-Net.Ru подразумевает, что вы прочитали и согласны с пользовательским соглашением http://www . mathnet.ru/rus/agreement

Параметры загрузки:

IP : 52.6 .47 .48

26 апреля 2023 г., 13:24:16

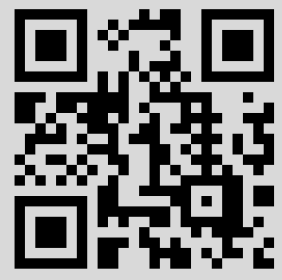




\section{Момент-угол многообразия простых $n$-мерных многогранников с $n+3$ гипергранями}

\section{Н. Ю. Ероховец}

В основе данной публикации лежит работа [1]. Пусть $P$ - простой выпуклый $n$-мерный многогранник и $\mathscr{F}=\left\{F_{1}, \ldots, F_{m}\right\}$ - множество его гиперграней. Каждой гиперграни $F_{i}$ сопоставим одномерную координатную подгруппу $T^{F_{i}} \subset T^{\mathscr{F}}=T^{m}$, грани $G$ сопоставим подгруппу $T^{G}=\prod_{F_{i} \supset G} T^{F_{i}}$. Момент-угол многообразие многогранника $P$ определяется как факторпространство $\mathscr{Z}_{P}=\left(T^{\mathscr{F}} \times P\right) / \sim$, где $\left(t_{1}, p\right) \sim$ $\left(t_{2}, q\right) \Leftrightarrow p=q, t_{1} t_{2}^{-1} \in T^{G(p)}, G(p)=\bigcap_{F_{i} \ni p} F_{i}$. Топологический тип пространства $\mathscr{Z}_{P}$ зависит только от комбинаторного типа многогранника $P$. Оно имеет структуру гладкого $(m+n)$-мерного многообразия с каноническим действием тора $T^{m}$, индуцированным очевидным действием тора $T^{m}$ на $T^{m} \times P$. Ясно, что $\mathscr{Z}_{P} / T^{m}=P$ и стабилизатором точки $[(t, q)]$ является $T^{G(q)}$. Числом Бухштабера $s(P)$ называется максимальная размерность подгрупп $H \cong T^{s}$, действующих свободно. Это число является комбинаторным инвариантом многогранника, причем $1 \leqslant s(P) \leqslant m-n$.

Проблема 1 (В. М. Бухштабер, 2002). Дать алгоритм вычисления инварианта $s(P)$ в терминах комбинаторики многогранника $P$.

$$
\text { Лемма 1. Пусть } \mathscr{F}=\bigcup_{i=1}^{r} \mathscr{F}_{i} \text {, где } \bigcap_{F_{j} \in \mathscr{F}_{i}} F_{j}=\varnothing . \text { Тогда } s(P) \geqslant m-\sum_{i=1}^{r}\left|\mathscr{F}_{i}\right|+r \text {. }
$$

Простой многогранник $P$ называется $k$-флаговым, если любой набор его гиперграней, из которых любые $k$ пересекаются, имеет непустое пересечение. Любой $n$-мерный многогранник, кроме симплекса $\Delta^{n}$, является $n$-флаговым.

Следствие 1. Пусть $P-k$-флаговый многогранник. Тогда $s(P) \geqslant\lceil(m-n) / k\rceil$. Рассмотрим биградуированную дифференциальную алгебру

$$
R^{*, *}(P)=\Lambda\left[u_{1}, \ldots, u_{m}\right] \otimes \mathbb{Z}[P] /\left(v_{i}^{2}=u_{i} v_{i}=0, i=1, \ldots, m\right),
$$

где bideg $u_{i}=(-1,2), \operatorname{bideg} v_{i}=(0,2), d u_{i}=v_{i}, d v_{i}=0$. В [2] доказан изоморфизм градуированных алгебр $\mathrm{H}^{*}\left(\mathscr{Z}_{P}\right) \simeq \mathrm{H}\left[R^{*}(P)\right]$, где $\operatorname{deg} x=-q+2 p$ для $x \in R^{-q, 2 p}(P)$. Он задает на $\mathrm{H}^{*}\left(\mathscr{Z}_{P}\right)$ структуру биградуированного кольца. Числа $\left\{\beta^{-q, 2 p}(P)=\right.$ $\left.\operatorname{rank} \mathrm{H}^{-q, 2 p}\left(\mathscr{Z}_{P}\right)\right\}$ являются комбинаторными инвариантами многогранника $P$ и называются биградуированными числами Бетти. Как известно, число минимальных наборов гиперграней, имеющих пустое пересечение, равно $l(P)=\sum_{p} \beta^{-1,2 p}(P)$.

Из теоремы 1 (см. ниже) мы получаем следующий результат по проблеме 1.

СлеДСтвие 2. Для многогранника $P^{n}$ с $m=n+3$ имеем: $s(P)=3$, если $l(P) \leqslant 7$, u $s(P)=2$, если $l(P)>7$. Таким образом, для многогранника с $m \leqslant n+3$ инвариант $s(P)$ выражается через биградуированные числа Бетти $\left\{\beta^{-q, 2 p}(P)\right\}$.

Известно (см. [3]), что любой простой многогранник $P$ с $m=n+3$ гипергранями комбинаторно описывается при помощи правильного $(2 k-1)$-угольника $M_{2 k-1}=$ $\operatorname{conv}\left\{\boldsymbol{w}_{r}=e^{2 \pi i r /(2 k-1)}, r \in \mathbb{Z}_{2 k-1}\right\}$ и следующего обобщения хроматического числа в виде сюръективного отображения $\zeta: \mathscr{F} \rightarrow \operatorname{vert}\left(M_{2 k-1}\right)$ такого, что для любой тройки $F_{r}, F_{s}, F_{t}$ дополнительные к ним гиперграни пересекаются в вершине

Работа выполнена при поддержке РФФИ (гранты № 10-01-92102-ЯФ-а, 11-01-00694-а), гранта Президента РФ МД-2253.2011.1 и гранта Правительства РФ 2010-220-01-077 (дог. 11.G34.31.005). 
тогда и только тогда, когда $0 \in \operatorname{conv}\left\{\zeta\left(F_{r}\right), \zeta\left(F_{s}\right), \zeta\left(F_{t}\right)\right\}$. Введем числа вершин $a_{r}=\left|\zeta^{-1}\left(\boldsymbol{w}_{r}\right)\right|$ и положим $P \sim\left(a_{1}, \ldots, a_{2 k-1}\right)$. Многогранники $P$ и $Q$ комбинаторно эквивалентны тогда и только тогда, когда существует ортогональное преобразование многоугольников, сохраняющее числа вершин. Для $k \geqslant 3$ любой многоугольник $\left(M_{2 k-1}, a_{1}, \ldots, a_{2 k-1}\right)$ реализуется, а для $k=2$ имеем $\Delta^{a} \times \Delta^{b} \times \Delta^{c} \sim(a+1, b+1, c+1)$, $a, b, c \geqslant 0$. Положим $\varphi_{r}=a_{r}+\cdots+a_{r+k-2}$ и $\psi_{r}=a_{r}+\cdots+a_{r+k-1}$.

ПредЛОЖениЕ 1 [4]. Пустъ $P \sim\left(a_{1}, \ldots, a_{2 k-1}\right)$. Тогда $s(P)=3$, если $k \leqslant 4$, $u s(P)=2$, если $k \geqslant 5$.

Teорема 1. Eсли $P \sim\left(a_{1}, \ldots, a_{2 k-1}\right)$, mo $^{*, *}\left(\mathscr{Z}_{P}\right)=\mathbb{Z} \oplus \mathbb{Z}^{2 k-1} \oplus \mathbb{Z}^{2 k-1} \oplus \mathbb{Z}-$ zpynna с образуюшими: $\left\{1, X_{i}, Y_{j}, Z: i, j=1, \ldots, 2 k-1\right\}$, $\operatorname{bideg} X_{i}=\left(-1,2 \varphi_{i}\right), \quad \operatorname{bideg} Y_{j}=$ $\left(-2,2 \psi_{j}\right)$, bideg $Z=(-3,2(n+3))$. Для $k=2$ имеем: $X_{i}^{2}=0, X_{i} X_{i+1}=Y_{i}$, $X_{1} X_{2} X_{3}=Z$. Для $k \geqslant 3$ имеем: $X_{i} X_{j}=0, X_{i} Y_{j}=\delta_{i+k-1, j} Z, Y_{i} Y_{j}=0$.

Доказательство опирается на изоморфизм $\mathrm{H}^{*}\left(\mathscr{Z}_{P}\right) \simeq \mathrm{H}\left[R^{*}(P)\right]$ и комбинаторику совокупности минимальных множеств непересекающихся гиперграней.

Следствие 3. Если $P \sim\left(a_{1}, \ldots, a_{2 k-1}\right), m o 2 k-1=\sum_{i} \beta^{-1,2 i}(P)=\sum_{j} \beta^{-2,2 j}(P)$.

Далее $P$ и $Q-$ многогранники с $m=n+3$.

СлЕДСтвиЕ 4. Имеем $\mathrm{H}^{*, *}\left(\mathscr{Z}_{P}\right) \simeq \mathrm{H}^{*, *}\left(\mathscr{Z}_{Q}\right)$ тогда и только тогда, когда биградуированные числа $\beta^{-q, 2 p}(P)=\beta^{-q, 2 p}(Q)$ равны для всех $p, q$.

Пример 1. Если $P \sim(a, a, \ldots, a), a \geqslant 1$, и $\beta^{-q, 2 p}(Q)=\beta^{-q, 2 p}(P)$ для всех $p, q$, то $Q=P$.

ПримеР 2. Если $P \sim\left(a_{1}, a_{2}, a_{3}, a_{4}, a_{5}\right)$ и $Q \sim\left(a_{1}, a_{2}, a_{4}+a_{5}-a_{2}, a_{2}+a_{3}-a_{5}, a_{5}\right)$, то $\mathrm{H}^{*, *}\left(\mathscr{Z}_{P}\right)=\mathrm{H}^{*, *}\left(\mathscr{Z}_{Q}\right)$. В случае $P \sim(1,1,2,2,2)$ и $Q \sim(1,1,3,1,2)$, например, многогранники комбинаторно не эквивалентны, но $\mathrm{H}^{*, *}\left(\mathscr{Z}_{P}\right)=\mathrm{H}^{*, *}\left(\mathscr{Z}_{Q}\right)$.

Известно (см. [4]), что в общем случае число $s(P)$ не определяется $f$-вектором $f(P)$ и хроматическим числом $\gamma(P)$ многогранника $P$. Например, для $P \sim(2,1,1,1,1,1$, $1,1,1)$ и $Q \sim(2,1,2,1,1,2,1)$ имеем: $f(P)=f(Q), \gamma(P)=\gamma(Q)$, но $s(P)=2, s(Q)=3$.

Следствие 2 приводит к вопросу: можсно ли вычислить инвариант $s(P)$ по биградуированным числам Бетти $\left\{\beta^{-q, 2 p}(P)\right\}$ при $m>n+3$ ?

Результат работы [5] о момент-угол комплексах позволяет связать теорему $1 \mathrm{c}$ результатом Лопе де Медрано [6] о полном пересечении квадрик. Пусть $k=2$. Тогда $\mathscr{Z}_{P}=S^{2 a+1} \times S^{2 b+1} \times S^{2 c+1}$, где $P \sim(a+1, b+1, c+1)$. Пусть $k \geqslant 3$. Тогда $\mathscr{Z}_{P}=\underset{i=1}{\#} S^{2 k-1} \times S^{2 \psi_{i+k-1}-2}$, где $P \sim\left(a_{1}, \ldots, a_{2 k-1}\right)$.

Автор благодарен В. М. Бухштаберу за постановку задачи и внимание к работе.

\section{Список литературы}

[1] N. Erokhovets, Buchstaber invariant of simple polytopes, arXiv: 0908.3407v1. [2] B. M. Бyxштабер, Т.Е. Панов, Торические действия в топологии и комбинаторике, МЦНМО, М., 2004. [3] B. Grunbaum, Convex polytopes, Springer, New York, 2003. [4] Н. Ю. Ероховец, YMH, 63:5 (2008), 187-188. [5] V. M. Buchstaber, T. E. Panov, N. Ray, Moscow Math. J., 7:2 (2007), 219-242; arXiv: 0609346. [6] S. López de Medrano, Lecture Notes in Math., 1370, 1989, $280-292$.

Н. Ю. Ероховец (N. Yu. Erokhovets)

Московский государственный университет им. М. В. Ломоносова

E-mail: erochovetsn@hotmail.com
Представлено В. М. Бухштабером Принято редколлегией 09.09.2011 